\title{
Independent prognostic value of angiogenesis and the level of plasminogen activator inhibitor type I in breast cancer patients
}

\author{
S Hansen*,', J Overgaard ${ }^{2}$, C Rose ${ }^{1,5}$, Ann Knoop', A-V Lænkholm', J Andersen ${ }^{3}$, FB Sørensen ${ }^{4}$ \\ and PA Andreasen ${ }^{6}$
}

'Department of Oncology, Oncological Research Centre, Odense University Hospital, DK-5000 Odense C, Denmark; ${ }^{2}$ Department of Experimental Clinical Oncology, Aarhus University Hospital, DK-8000 Aarhus C, Denmark; ${ }^{3}$ Department of Oncology, Aarhus University Hospital, DK-8000 Aarhus C, Denmark; ${ }^{4}$ Department of Pathology, Aarhus University Hospital, DK-8000 Aarhus C, Denmark; ${ }^{5}$ Department of Oncology, Lund University Hospital, S-22I 85 Lund, Sweden; ${ }^{6}$ Department of Molecular and Structural Biology, Aarhus University, DK-8000 Aarhus C, Denmark

Tumour angiogenesis and the levels of plasminogen activator inhibitor type I (PAI-I) are both informative prognostic markers in breast cancer. In cell cultures and in animal model systems, PAI-I has a proangiogenic effect. To evaluate the interrelationship of angiogenesis and the PAI-I level in breast cancer, we have evaluated the prognostic value of those factors in a total of 228 patients with primary, unilateral, invasive breast cancer, evaluated at a median follow-up time of 12 years. Microvessels were immunohistochemically stained by antibodies against CD34 and quantitated by the Chalkley counting technique. The levels of PAI-I and its target proteinase UPA in tumour extracts were analysed by ELISA. The Chalkley count was not correlated with the levels of uPA or PAI-I. High values of UPA, PAI-I, and Chalkley count were all significantly correlated with a shorter recurrence-free survival and overall survival. In the multivariate analysis, the uPA level did not show independent prognostic impact for any of the analysed end points. In contrast, the risk of recurrence was independently and significantly predicted by both the PAI-I level and the Chalkley count, with a hazard ratio $(95 \% \mathrm{Cl})$ of $1.6(1.01-2.69)$ and $1.4(1.02-1.81)$, respectively. For overall survival, the Chalkley count, but not PAI-I, was of significant independent prognostic value. The risk of death was I.7 (I.30-2. I5) for Chalkley counts in the upper tertile compared to the lower one. We conclude that the PAI-I level and the Chalkley count are independent prognostic markers for recurrence-free survival in patients with primary breast cancer, suggesting that the prognostic impact of PAI-I is not only based on its involvement in angiogenesis.

British Journal of Cancer (2003) 88, 102 -108. doi:10.1038/sj.bjc.6600662 www.bjcancer.com

(c) 2003 Cancer Research UK

Keywords: breast-neoplasms; neovascularization; prognosis; survival-analysis

Tumour growth can be dependent on angiogenesis, that is, the formation of new blood vessels from the existing capillary network (Folkman, 1971, 1995). Tumour progression seems to be dependent on cancer cell controlled tissue remodelling, including angiogenesis, mediated to a large extent by the plasminogen activation system (Mignatti and Rifkin, 1996; Pepper et al, 1996; Mazar et al, 1999). The hypothesis of angiogenesis as a prognosticator has been widely investigated using different assays for determining microvessel density (Fox, 1997; Hansen et al, 2000a). Angiogenesis determined by Chalkley counting has shown independent prognostic value in a large population-based study of primary breast cancer patients (Hansen et al, 2000b). Breast cancer patients whose tumours had a Chalkley count in the upper tertile had, compared to patients belonging to the lowest tertile, a $126 \%$ increased risk of dying and patients in the middle tertile a $55 \%$ increased risk (Hansen et al, 2000b). Likewise, the urokinase-type (uPA) plasminogen activator system has been shown to play a crucial role in cancer metastasis (Andreasen et al, 1997, 2000).

*Correspondence: Dr S Hansen; E-mail: steinbjoern.hansen@dadlnet.dk Received 22 May 2002; revised 16 September 2002; accepted 19 September 2002
Various components of the uPA system, including uPA itself and its primary inhibitor, plasminogen activator inhibitor type 1 (PAI1), have demonstrated significant prognostic impact in breast cancer patients. Thus, the level of PAI-1 in primary tumours is one of the most informative biochemical prognostic markers in several cancer types (Duffy, 1996; Harbeck et al, 1998; Knoop et al, 1998; Duffy et al, 1999; Look and Foekens, 1999; Janicke et al, 2001; Look et al, 2002).

In several cell culture and animal model systems, PAI-1 has been found to have a proangiogenic effect (Bajou et al, 1998, 2001; Lambert et al, 2001; McMahon et al, 2001; Devy et al, 2002). However, the clinical significance of the biological interaction of angiogenesis and the plasminogen activator system is unknown. Although the prognostic impacts of the Chalkley count and PAI-1 have been reported individually in large study populations, it is highly relevant to evaluate the combined prognostic impact of angiogenesis and the levels of the components of the plasminogen activator system in the same study population.

The aims of the present study were to evaluate the association between the Chalkley count, the levels of PAI-1 and of uPA, and the independent prognostic value of these components in relation to the classical prognostic factors in breast cancer. 


\section{MATERIAL AND METHODS}

\section{Patients}

The study included 228 patients who underwent surgery for primary, unilateral, invasive breast carcinoma. Inclusion was restricted to patients referred from the primary catchment area of Odense University Hospital during the period from 1 August 1984 to 1 September 1989. Mammographic screening for breast cancer was not performed in the background population during this period. Excluded were patients with distant metastasis at the time of diagnosis, locally advanced disease, inflammatory carcinoma, synchronous bilateral breast cancer, and a diagnosis of isolated carcinoma in situ. Women with previous malignant disease, apart from carcinoma in situ of the uterine cervix or nonmelanotic skin cancer, were excluded, as were women who did not undergo axillary dissection with at least one lymph node removed. The analysis was restricted to those 228 patients who had residual tumour material stored at $-80^{\circ} \mathrm{C}$ at the time of surgery. Earlier we reported the Chalkley counts on a population-based cohort of 836 patients (Hansen et al, 2000b). The 228 patients are a subgroup of this cohort, sampled from a shorter period of time (432 patients), and further restricted to patients having frozen tumour material available. Also, we have earlier reported on uPA and PAI-1 from a larger cohort of 429 patients based on frozen tumour samples (Knoop et al, 1998). Again, the 228 patients are a subgroup of this cohort restricted to the primary catchment area of Odense University Hospital.

\section{Treatment}

Surgery, radiotherapy, and adjuvant systemic therapy were carried out according to the nationwide recommendation of the Danish Breast Cancer Cooperative Group (DBCG) (Andersen and Mouridsen, 1988). In all, 27 patients were treated with breast-conserving surgery and all had postoperative irradiation. In total, 201 patients underwent simple mastectomy, and of these 63 had postoperative irradiation. High-risk patients (N1, T3 or T4) were offered systemic adjuvant therapy according to the DBCG programme (Fischerman and Mouridsen, 1988). In summary, 42 patients had primary chemotherapy (CMF), 50 patients primary endocrine therapy (tamoxifen), and 12 both endocrine therapy and chemotherapy. A total of 124 patients received no systemic treatment: of these 82 were considered low-risk (N0, T1-2) patients, while 42 patients were either too old or were found to have some medical contraindications for systemic treatment.

\section{Follow-up}

Patients were followed up on a regular basis for the first 10 years at Odense University Hospital according to the DBCG recommendations (Andersen and Mouridsen, 1988). After the first 10 years, information about recurrence was obtained by studying the clinical records from the treating department. Patients were followed until the time of the last contact or death, or until the closing date of the study, 1 March 1999. The median follow-up time was 12 years and $1 / 2$ month.

\section{End points}

The prognostic analysis was primarily carried out using recurrence-free survival (RFS) and overall survival (OS). The RFS analysis was based on time to first recurrence at any site including 89 events. Thus, patients who died without recurrence were censored. The OS analysis was based on death from any cause comprising 118 events.

\section{Histopathology}

The histological type of breast tumour was determined according to the WHO guidelines (Scharff and Torloni, 1998). Histological malignancy grading followed the grading system of Bloom and Richardson (1957). Tumour size was measured as the largest diameter of the invasive carcinoma. Receptor status was retrieved from the DBCG database and considered positive if the estrogen receptor (ER) or progesterone receptor $(\mathrm{PgR})$ value by the dextran-coated charcoal (DCC) method was greater than or equal to $10 \mathrm{fmol} \mathrm{mg}^{-1}$ cytosol protein. Where the DCC method was unavailable, a positive receptor status was determined by an immunohistochemical ER staining (antibody clone 1D5, DAKO, Glostrup, Denmark). ER-positive was defined as $\geqslant 10 \%$ of the tumour cells staining positively.

\section{Angiogenesis}

The estimation of angiogenesis by Chalkley counts has been described in detail earlier (Hansen et al, 2000b). Briefly, one $4 \mu \mathrm{m}$ thick section from each formalin-fixed and paraffin-embedded tumour was mounted on a ChemMate slide (DAKO, Glostrup, Denmark). Epitope retrieval was performed by microwave heating in a buffer of $10 \mathrm{~mm}$ Tris ( $\mathrm{pH}$ 9), $0.5 \mathrm{~mm}$ EGTA. As primary antibody against CD34, we used clone QBEnd/10 (Novo Castra, Newcastle, UK), diluted $1: 20$, with overnight incubation at $4{ }^{\circ} \mathrm{C}$.

The angiogenesis assessed by the Chalkley count technique was based on scanning the tumour section at low magnification and choosing three of the most vascularised areas (hot-spots), which were assumed to have the highest number of microvessel profiles. Using a higher magnification, a 25-point Chalkley graticule was applied to each hot-spot and oriented to permit the maximum number of points to hit the immunohistochemically highlighted microvessel profiles (Leitz Ortoplane; $\times 250$; Chalkley grid area $0.196 \mathrm{~mm}^{2}$ ). The Chalkley count was taken as the mean value of the three counts for each tumour. The Chalkley count is given as the number of hitting points without a unit; measurement range $0-25$.

\section{uPA and PAI-1}

The antigen levels of uPA and PAI-1 were assessed with ELISA as described in detail earlier (Knoop et al, 1998). Briefly, tissue for analysis was taken from $-80^{\circ} \mathrm{C}$, homogenised in a buffer of $0.1 \mathrm{~mm}$ Tris (pH 8.1), $0.5 \%$ Triton X-100, $10 \mathrm{~mm} \mathrm{EDTA,} 10 \mu \mathrm{g} \mathrm{ml}^{-1}$ aprotinin ( $10 \mu \mathrm{l}$ buffer per $\mathrm{mg}$ tissue), and centrifuged. The supernatants were analysed. For the uPA ELISA, monoclonal antiuPA IgG from hybridoma clones 2, 6, and 12 was used on the solid phase. For the PAI-1 ELISA, monoclonal anti-PAI-1 IgG from hybridoma clone 2 was used on the solid phase. The second antibody layer consisted of polyclonal rabbit anti-uPA or rabbit anti-PAI-1 antibodies. As the third layer, we used peroxidaseconjugated swine antibodies against rabbit antibodies for both ELISAs. The levels of uPA or PAI-1 were expressed as ng per mg total protein in the extracts.

\section{Statistics}

Angiogenesis, PAI-1, and uPA were estimated without knowledge of the clinical data or prognostic outcome. Predetermined cutoff points from the earlier studies (Knoop et al, 1998; Hansen et al, 2000b) were used for the prognostic analysis: Chalkley count at 5 and 7 points, PAI- 1 at $11.1 \mathrm{ng} \mathrm{mg}^{-1}$ total protein, and uPA at $4.5 \mathrm{ng} \mathrm{mg}^{-1}$ total protein. The relationship between the Chalkley count, the PAI-1 level, and the uPA level were tested by the Spearman correlation test. The possible associations of the classical clinical pathological parameters on one side and the Chalkley count, the PAI-1 level, and the uPA level on the other 
Table I Description of clinicopathological data and the association with the Chalkley count, PAI-I levels and uPA levels

\begin{tabular}{|c|c|c|c|c|c|}
\hline Variables & $\mathbf{N}$ & (\%) & $\begin{array}{l}\text { Chalkley count } \\
\text { (points) } \\
\text { Median }\end{array}$ & $\begin{array}{c}\text { PAI-I } \\
\left(\mathrm{ng} \mathrm{mg}^{-1} \text { total protein }\right) \\
\text { Median }\end{array}$ & $\begin{array}{c}\text { uPA } \\
\text { (ng } \mathrm{mg}^{-1} \\
\text { total protein) } \\
\text { Median }\end{array}$ \\
\hline All & 228 & $(100)$ & 6.0 & 11.05 & 4.65 \\
\hline \multicolumn{6}{|l|}{ Age (years) } \\
\hline$<40$ & 11 & (5) & 6.3 & 11.4 & 4.2 \\
\hline $40-49$ & 41 & $(18)$ & 6.1 & 10.7 & 3.3 \\
\hline $50-59$ & 51 & (22) & 6.0 & 10.1 & 5.0 \\
\hline $60-69$ & 60 & (26) & 6.3 & 11.3 & 4.9 \\
\hline \multirow{2}{*}{$\geqslant 70$} & 65 & (29) & 5.7 & 11.3 & 5.5 \\
\hline & & & $P=0.99$ & $P=0.90$ & $P=0.69$ \\
\hline \multicolumn{6}{|l|}{ Menopausal status } \\
\hline Pre & 68 & (30) & 6.2 & 10.6 & 4.2 \\
\hline \multirow[t]{2}{*}{ Post } & 160 & (70) & 6.0 & 11.3 & 5.0 \\
\hline & & & $P=0.86$ & $P=0.31$ & $P=0.27$ \\
\hline \multicolumn{6}{|l|}{ Tumour size (mm) } \\
\hline$\leqslant 20$ & 86 & (38) & 5.7 & 10.5 & 3.8 \\
\hline $21-50$ & 132 & (58) & 6.3 & 12.7 & 5.7 \\
\hline \multirow{2}{*}{$>50$} & 10 & (4) & 6.5 & 8.1 & 3.0 \\
\hline & & & $P=0.004$ & $P=0.019$ & $P=0.004$ \\
\hline \multicolumn{6}{|c|}{ Histological malignancy grade } \\
\hline I (ductal) & 36 & $(16)$ & 5.2 & 9.6 & 4.4 \\
\hline ॥ (ductal) & 87 & (38) & 6.0 & 12.1 & 5.4 \\
\hline III (ductal) & 74 & (32) & 7.0 & 12.9 & 5.5 \\
\hline \multirow{2}{*}{ Other (nonductal) } & 31 & (14) & 5.1 & 5.1 & 1.8 \\
\hline & & & $P<0.001$ & $P<0.001$ & $P<0.00 I$ \\
\hline \multicolumn{6}{|l|}{ Receptor status } \\
\hline Positive & 174 & (76) & 5.7 & 10.8 & 4.5 \\
\hline \multirow{2}{*}{ Negative } & 54 & (24) & 7.7 & 12.9 & 5.6 \\
\hline & & & $P<0.001$ & $P=0.027$ & $P=0.069$ \\
\hline \multicolumn{6}{|l|}{ Lymph node metastasis } \\
\hline None & 101 & (44) & 6.0 & 9.7 & 4.2 \\
\hline $1-3$ & 78 & (34) & 6.0 & 12.5 & 5.4 \\
\hline \multirow[t]{2}{*}{$\geqslant 4$} & 49 & (22) & 6.7 & 14.7 & 5.2 \\
\hline & & & $P=0.82$ & $P=0.008$ & $P=0.055$ \\
\hline
\end{tabular}

Significant associations are given in bold.

side were tested by the Kruskall-Wallis test. The univariate relationship between prognostic variables and the follow-up end points was illustrated by Kaplan-Meier plots of survival probabilities (Kaplan and Meier, 1958), and the survival functions were compared by the log-rank test. The multivariate relationship was evaluated by the Cox proportional hazard regression analysis (Cox, 1972). To obtain acceptable statistical strength in the multivariate analysis, it has been suggested that the number of events should be at least 10 times the total number of variables included (Harrell et al, 1985; Simon and Altman, 1994; Peduzzi et al, 1996). In this study, with a limited number of events, it is not advisable to introduce many covariates, and this was the reason for not estimating the prognostic effect of each subgroup of the variable. Therefore, the risk estimates for each variables are an average between subgroups; that is, lowest to middle to highest tertile. Thus, using eight variables in our Cox models, we assumed that we could achieve a reliable statistical strength of the risk estimates in the multivariate regression analysis. As in the earlier publication (Knoop et al, 1998), the Cox models included the classical prognostic factors (menopausal status, tumour size, malignancy grade, receptor status, and number of positive nodes) together with uPA, PAI-1, and the Chalkley count, in that order. The Cox models were stratified by receptor status, which did not fulfil the assumption of proportional hazard rates. Two-sided $P$-values below 0.05 were considered to be statistically significant.

\section{RESULTS}

\section{Clinical data}

The tertiles of the Chalkley estimates were 5.3 and 7.0 points, of the PAI-1 levels 8.1 and $15.1 \mathrm{ng} \mathrm{mg}^{-1}$ total protein, and of the uPA levels 3.2 and $6.3 \mathrm{ng} \mathrm{mg}^{-1}$ total protein. The median (range) of the Chalkley count was $6.0(2.7-13.0)$, of PAI-1 $11.1 \mathrm{ng} \mathrm{mg}^{-1}$ $(0.1-73.0)$, and of $\mathrm{uPA}$ of $4.7 \mathrm{ng} \mathrm{mg}^{-1}(0.3-21.6)$. Table 1 shows the distributions of the clinical and pathological data of the patients in the study population and the distribution of the Chalkley count, the PAI-1 level, and the uPA level. A high Chalkley count was significantly associated with large tumour size, high histological malignancy grade, negative receptor status, but not with a high number of axillary lymph node metastases, age, or menopausal status. A high PAI-1 level was significantly associated with large tumour size, high histological malignancy grade, negative receptor status, and a high number of axillary lymph node metastases, 
but not with age or menopausal status. A high uPA level was significantly associated with large tumour size, high histological malignancy grade, and marginally significantly associated with negative receptor status and a high number of positive axillary lymph node metastases, but not with age or menopausal status.

\section{Association}

Figure 1 shows scatter plots illustrating the relationship between the Chalkley count, the PAI-1 level, and the uPA level. High levels of UPA were weakly linearly associated with high levels of PAI-1 $(r=0.57, P<0.001)$. In contrast to this, there was no indication of an association between the Chalkley count and PAI-1 or uPA levels in primary breast carcinomas.

\section{Univariate analysis}

The Kaplan-Meier plots in Figure 2 illustrate the significantly poorer prognostic outcome for patients with high Chalkley counts $(P=0.0029$, RFS; $P<0.0001$, OS $)$, high PAI-1 levels $(P=0.0001$, RFS; $P=0.0004$, OS), and high uPA levels $(P=0.0328$, RFS; $P=0.0354$, OS). The 5-year-survival probabilities for the three investigated factors as well as the classical prognostic factors in breast cancer are tabulated in Table 2. Among the classical prognostic factors, the univariate analysis showed a significantly poorer prognostic outcome for patients with large tumour size $(P=0.0004$, RFS; $P=0.0002$, OS $)$, high histological malignancy grade $(P=0.0061$, RFS; $P=0.0007$, OS), high number of positive axillary lymph nodes $(P<0.0001$ for both RFS and OS), while postmenopausal patients had a significantly poorer outcome only for OS $(P=0.011)$.

\section{Multivariate analysis}

Table 3 shows the results of the Cox multivariate regression analysis including the classical prognostic factors (menopausal status, tumour size, histological malignancy grade, receptor status, and number of axillary lymph node metastases) as well as the levels of uPA and PAI-1 and the Chalkley count, which were introduced in the model in that order. The cutoff values from Table 1 were used for the classical factors, while the cutoff values for the levels of uPA and PAI-1 and the Chalkley count were used as defined in earlier studies (Knoop et al, 1998; Hansen et al, $2000 \mathrm{~b})$. The nonductal carcinomas were grouped with ductal malignancy grade II carcinomas because they had approximately the same survival. Using the recurrence-free survival as an endpoint, there was significant independent information from tumour size, lymph node metastasis, PAI-1, and Chalkley count. There was a $70 \%$ increased risk of recurrence $(\mathrm{HR}=1.7$ (1.022.72)) for patients with PAI-1 values above the median as compared to values below the median. There was a $40 \%$ increased risk of recurrence $(\mathrm{HR}=1.4(1.03-1.84))$ for patients with Chalkley counts in the middle tertiles as compared to the lowest tertile, and a further $40 \%$ increase in the risk of recurrence in having a Chalkley count in the upper tertile as compared to the middle tertile. Analysing overall survival, menopausal status, tumour size, malignancy grade, lymph node metastasis, and Chalkley counts showed a significant independent prognostic value. There was a $70 \%$ increase in the risk of dying $(\mathrm{HR}=1.7$ $(1.32-2.20))$ for patients with Chalkley counts going from one tertile to the next.

\section{DISCUSSION}

Angiogenesis and extracellular proteolysis of the plasminogen activation system are of crucial importance in cancer metastasis (Andreasen et al, 1997). A clinical usefulness of these systems to
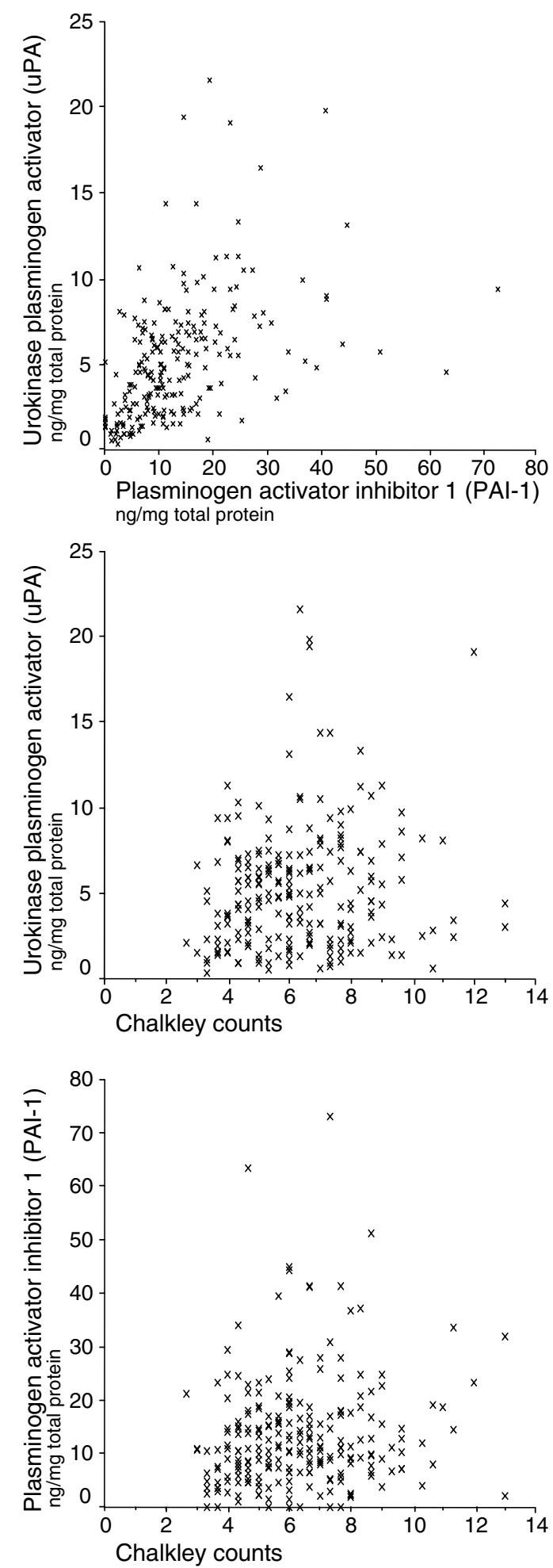

Figure I Relationships between the levels of UPA, PAI-I, and the Chalkley count.

predict the outcome in breast cancer patients has been expected. Numerous studies have established a prognostic value of the levels of UPA and PAI-1 and of angiogenesis in breast cancer patients (Fox, 1997; Harbeck et al, 1998; Duffy et al, 1999; Look and Foekens, 1999; Look et al, 2002). However, none of these factors are yet used in current clinical practice. 

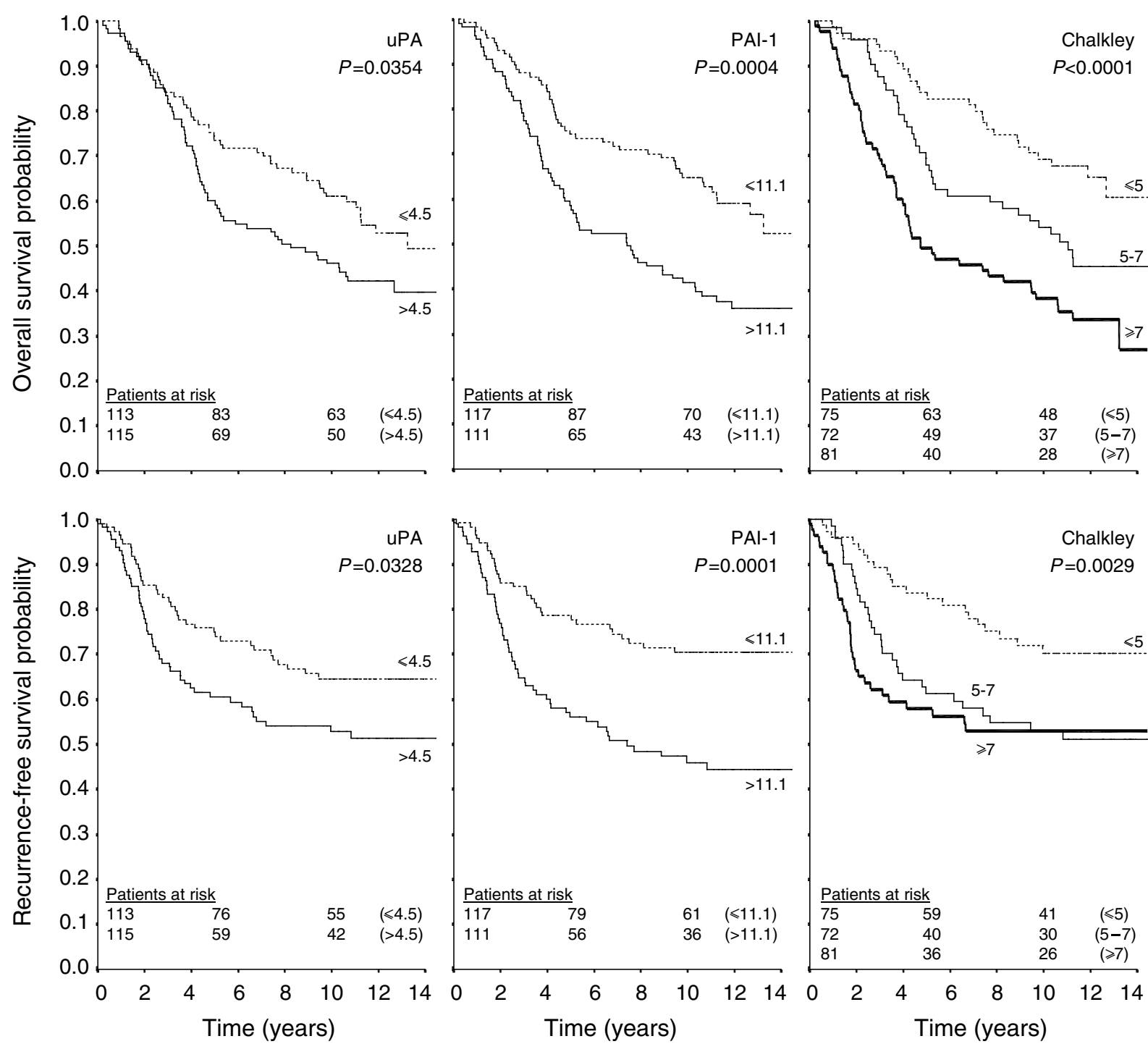

Figure 2 Kaplan-Meier plots of the survival probabilities for the different categories of the Chalkley count, the uPA level, and the PAI-I level. The survival curves in the upper row give the probabilities of overall survival, and in the bottom row the probabilities of recurrence-free survival. Predefined cutoff values (see Statistics section) were $4.5 \mathrm{ng} \mathrm{mg}^{-1}$ total protein for the uPA level, II.I ng mg ${ }^{-1}$ total protein for the PAl-I level, and 5 and 7 points for the Chalkley count. The number of patients at risk in each group is given at $0-, 5-$, and 10 -years follow-up.

The biological interpretation of the prognostic impact of high values of uPA and of angiogenesis has been straightforward. A high number of vessel profiles should increase the nourishment and growth of the tumour and allow access of tumour cells to the circulation. High uPA levels facilitate the proteolytic degradation of the basement membranes and extracellular matrix, and thereby increase the invasive ability and metastatic potential. Moreover, angiogenesis is believed to require extracellular proteolytic activity, including uPA activity (Mignatti and Rifkin, 1996; Pepper et al, 1996; Mazar et al, 1999). The prognostic impact of high levels of PAI-1 has been more difficult to explain. Generally, we could expect a requirement for the presence of proteinase inhibitors during tissue remodelling events, because of a need to restrict proteolysis in time and space. Hence, the association of high PAI-1 levels with a poor prognostic outcome can be explained by a requirement for local downregulation of the proteolytic activity. In particular, proteolytic downregulation could support a local protection of the basement membrane surrounding the sprouting endothelial cells. This could further facilitate the capillary networking of the new tumour blood vessels. Accordingly, it has been reported that tumour angiogenesis is drastically impaired in mice with targeted disruption of the PAI-1 gene (Bajou et al, 1998, 2001). A few other model system studies have also suggested a proangiogenic role of PAI-1 (Lambert et al, 2001; McMahon et al, 2001; Devy et al, 2002). In particular, the possible role of uPA and PAI-1 in angiogenesis was of interest in the preparation of the present study. Owing to the above explanations, we had reasons to expect biological relations between levels of the components of the plasminogen activating system, especially PAI-1, and the angiogenic process. One might expect PAI-1 to be a surrogate marker for the angiogenic activity. This could be expected to be seen as a positive association between PAI-1 and the Chalkley count, and possibly a prognostic value of PAI-1 and the Chalkley count being dependent on each other.

In general, the estimates of the Chalkley count, PAI-1, and uPA from this study are in agreement with our earlier reports (Knoop et al, 1998; Hansen et al, 2000b). In the present study, the Chalkley count was not associated with the levels of uPA or PAI-1. This is in accordance with another report (Fox et al, 2001) showing no association between the Chalkley count and uPA or PAI-1. 
Table 2 Five-year survival probabilities \pm s.e. in percentages for recurrence-free survival (RFS), and overall survival (OS)

\begin{tabular}{|c|c|c|c|}
\hline Variables & $\mathbf{N}$ & $\begin{array}{c}\text { RFS } \\
\%( \pm \text { s.e. })\end{array}$ & $\begin{array}{c}\text { OS } \\
\%( \pm \text { s.e. })\end{array}$ \\
\hline All & 228 & $68(3)$ & $67(3)$ \\
\hline \multicolumn{4}{|l|}{ Menopausal status } \\
\hline Pre & 68 & $67(6)$ & $74(5)$ \\
\hline \multirow[t]{2}{*}{ Post } & 160 & $68(4)$ & $64(4)$ \\
\hline & & $P=0.926$ & $P=0.011$ \\
\hline \multicolumn{4}{|l|}{ Tumour size (mm) } \\
\hline$\leqslant 20$ & 86 & $83(4)$ & $81(4)$ \\
\hline $21-50$ & 132 & $59(4)$ & $59(4)$ \\
\hline \multirow[t]{2}{*}{$>50$} & 10 & $40(15)$ & $40(15)$ \\
\hline & & $P=0.0004$ & $P=0.0002$ \\
\hline \multicolumn{4}{|c|}{ Histological malignancy grade } \\
\hline I (ductal) & 36 & $88(5)$ & $92(5)$ \\
\hline II (ductal) & 87 & $66(5)$ & $69(5)$ \\
\hline III (ductal) & 74 & $55(6)$ & $49(6)$ \\
\hline \multirow[t]{2}{*}{ Other (nonductal) } & 31 & $76(8)$ & $74(8)$ \\
\hline & & $P=0.006 I$ & $P=0.0007$ \\
\hline \multicolumn{4}{|l|}{ Receptor status } \\
\hline Positive & 174 & $69(4)$ & $68(4)$ \\
\hline \multirow[t]{2}{*}{ Negative } & 54 & $64(7)$ & $63(7)$ \\
\hline & & $P=0.88 \mid$ & $P=0.857$ \\
\hline \multicolumn{4}{|c|}{ Lymph node metastasis } \\
\hline None & 101 & $75(4)$ & $77(4)$ \\
\hline $1-3$ & 78 & 78 (5) & $72(5)$ \\
\hline \multirow[t]{2}{*}{$\geqslant 4$} & 49 & $37(7)$ & $37(7)$ \\
\hline & & $P<0.0001$ & $P<0.0001$ \\
\hline \multicolumn{4}{|c|}{ Chalkley count (points) } \\
\hline$\leqslant 5$ & 75 & $84(4)$ & $84(4)$ \\
\hline $5-7$ & 72 & $61(6)$ & $68(5)$ \\
\hline \multirow[t]{2}{*}{$\geqslant 7$} & 81 & $58(6)$ & $49(6)$ \\
\hline & & $P=0.0029$ & $P<0.0001$ \\
\hline \multicolumn{4}{|c|}{ PAl-I (ng mg ${ }^{-1}$ total protein) } \\
\hline$\leqslant 11.1$ & 117 & $79(4)$ & $74(4)$ \\
\hline \multirow{2}{*}{$>11.1$} & |11 & $56(5)$ & $59(5)$ \\
\hline & & $P=0.0001$ & $P=0.0004$ \\
\hline \multicolumn{4}{|c|}{ uPA (ng mg ${ }^{-1}$ total protein) } \\
\hline$\leqslant 4.5$ & 113 & $75(4)$ & $73(4)$ \\
\hline \multirow[t]{2}{*}{$>4.5$} & 115 & $60(5)$ & $60(5)$ \\
\hline & & $P=0.0328$ & $P=0.0354$ \\
\hline
\end{tabular}

Significant differences in survival probabilities between the groups are given in bold.

However, that study did not evaluate the independent prognostic value of the Chalkley count in relation to the UPA or PAI-1 levels, because of an 'insufficient number of events for the number of variables' (Fox et al, 2001). Others have reported a moderate association between angiogenesis and uPA $(r=0.85)$, as well as PAI-1 $(r=0.74)$ (Hildenbrand et al, 1995). This might be explained from the fewer $(n=42)$ patients investigated in those studies, from correlating uPA from the periphery of the tumour specimen with the angiogenesis, and from estimating angiogenesis by the microvessel density method. We chose the Chalkley count estimate of angiogenesis, since this assay is somewhat less influenced by the observer variation (Hansen et al, 1998), and because it has a stronger prognostic value as compared to the microvessel density assay (Rose et al, 2000). The frozen tumour specimens used for the uPA and PAI-1 analyses do not represent the same tumour areas as the paraffin-embedded specimens used for the Chalkley counts.
Table 3 The Cox multivariate analysis estimated the hazard ratios (HR) and $95 \% \mathrm{Cl}$ for the risk of recurrence (RFS) and risk to die (OS) in a group of 228 patients with breast cancer

\begin{tabular}{|c|c|c|c|c|c|c|}
\hline \multirow[b]{2}{*}{ Variables } & \multicolumn{3}{|c|}{ RFS } & \multicolumn{3}{|c|}{ os } \\
\hline & $P$ & HR & $(95 \% \mathrm{Cl})$ & $P$ & HR & $(95 \% \mathrm{Cl})$ \\
\hline Menopausal status & 0.91 & 1.0 & $(0.61-1.55)$ & 0.003 & 2.0 & $(1.26-3.12)$ \\
\hline Tumour size & 0.015 & 1.7 & $(1.10-2.57)$ & 0.014 & 1.6 & $(1.10-2.23)$ \\
\hline Malignancy grade & 0.081 & 1.4 & $(0.96-1.93)$ & 0.011 & 1.5 & $(1.10-2.09)$ \\
\hline Lymph node status & $<0.0001$ & 1.9 & $(1.40-2.50)$ & $<0.0001$ & 2.0 & $(1.53-2.52)$ \\
\hline UPA & 0.33 & 1.3 & $(0.79-2.01)$ & 0.60 & 1.1 & $(0.74-1.67)$ \\
\hline PAI-I & 0.040 & 1.7 & $(1.02-2.72)$ & 0.28 & 1.3 & $(0.83-1.92)$ \\
\hline Chalkley count & 0.032 & 1.4 & $(1.03-1.84)$ & $<0.0001$ & 1.7 & $(1.32-2.20)$ \\
\hline
\end{tabular}

The variables were grouped as given in Table 2. The nonductal carcinomas were included in the group of grade II ductal carcinomas. The Cox models were stratified by oestrogen receptor status, which did not fulfill the assumption of proportional hazard rates. Bold $P$-values are given for variables with hazard ratios significantly different from one.

Owing to the tumour heterogeneity, the tumour values of biologically related factors obtained from estimates in different regions may not be associated, although measurements of the factors obtained from the same areas would be associated. The levels of PAI-1 and uPA were low in very large tumours ( $>50 \mathrm{~mm}$ ) and in nonductal carcinomas. Hence, it is necessary to be aware of possible sampling errors, which in large tumours could be affected by necrotic areas, and in lobular carcinomas by the lower cellularity compared to the ductal carcinomas.

The independent prognostic effect of the Chalkley count was approximately of the same magnitude as reported earlier (Fox et al, 1995; Hansen et al, 2000b). In accordance with our earlier findings (Knoop et al, 1998), the uPA did not reveal any independent prognostic value. The PAI-1 estimate did have independent prognostic impact regarding the risk of recurrence as in our earlier report (Knoop et al, 1998). The PAI-1 estimate did not provide a significant independent prognostic value for the risk of death in the present smaller sample of the former population. This was also the case in a Cox model not including the Chalkley count (data not shown). We stratified the Cox multivariate models by receptor status, because it does not fulfil the assumption of proportional hazards. Hence, the independent prognostic estimates of uPA, PAI1 and the Chalkley count are adjusted for the effect of the receptor status as well as the other classical prognostic factors included.

The lack of correlation between the level of PAI-1 and the Chalkley count at first sight appears in contradiction to the hypothesis of PAI-1 being implicated in angiogenesis, as discussed above. Several explanations may be offered for the lack of correlation. First, the angiogenesis may be regulated differently in the human breast tumours studied here and in the experimental models discussed above. Second, if PAI-1 is implicated in the angiogenic process, it may only be transiently expressed and its level may therefore be associated with the rate of vessel formation rather than the accumulated amount of vessels, represented by the Chalkley count (Fox et al, 2001). Third, it is because of heterogeneity of the level of PAI-1 and the Chalkley count within the tumour, as discussed above. Fourth, PAI-1 may have a multitude of functions in breast tumours, angiogenesis only being one among several. The latter hypothesis is favoured by the immunohistochemical localization of PAI-1 to several cell types in breast tumours, including not only endothelial cells but also fibroblasts and cancer cells (Christensen et al, 1996).

The major finding in our present investigation is the independent prognostic impact of both the PAI-1 and the Chalkley count in the same primary breast tumours. This fits well with the 
lack of association between these estimates, which may independently contribute to the regulation of tumour progression. In conclusion, both PAI-1 and the Chalkley count added significant and independent prognostic information on RFS in patients with primary breast cancer.

\section{ACKNOWLEDGEMENTS}

The technical assistance of Ole Nielsen is greatly appreciated. We thank Dr Susan M Thorpe for providing us with her results from the analysis of the estrogen and progesterone receptors.

This study was supported by grants from the Danish Cancer Society, the Danish Medical Research Council, and Odense University.

\section{REFERENCES}

Andersen KW, Mouridsen HT (1988) Danish Breast Cancer Cooperative Group (DBCG). A description of the register of the nation-wide programme for primary breast cancer. Acta Oncol 27: 627-647

Andreasen PA, Egelund R, Petersen HH (2000) The plasminogen activation system in tumor growth, invasion, and metastasis. Cell Mol Life Sci 57: $25-40$

Andreasen PA, Kjoller L, Christensen L, Duffy MJ (1997) The urokinasetype plasminogen activator system in cancer metastasis: a review. Int $J$ Cancer 72: 1-22

Bajou K, Masson V, Gerard RD, Schmitt PM, Albert V, Praus M, Lund LR, Frandsen TL, Brunner N, Dano K, Fusenig NE, Weidle U, Carmeliet G, Loskutoff D, Collen D, Carmeliet P, Foidart JM, Noel A (2001) The plasminogen activator inhibitor PAI-1 controls in vivo tumor vascularization by interaction with proteases, not vitronectin. Implications for antiangiogenic strategies. J Cell Biol 152: 777-784

Bajou K, Noel A, Gerard RD, Masson V, Brunner N, Holst-Hansen C, Skobe M, Fusenig NE, Carmeliet P, Collen D, Foidart JM (1998) Absence of hos plasminogen activator inhibitor 1 prevents cancer invasion and vascularization. Nat Med 4: 923-928

Bloom HJG, Richardson WW (1957) Histological grading and prognosis in breast cancer. A study of 1409 cases of which 359 have been followed for 15 years. Br J Cancer 11: 359-377

Christensen L, Simonsen ACW, Heegaard CW, Moestrup SK, Andersen JA, Andreasen P (1996) Immunohistochemical localization of urokinase-type plasminogen activator, type-1 plasminogen-activator inhibitor, urokinase receptor and alpha-2-macroglobulin receptor in human breast carcinomas. Int J Cancer 66: 441-452

Cox DR (1972) Regression models and life tables. J Roy Stat Soc 34: 187-220

Devy L, Blacher S, Grignet-Debrus C, Bajou K, Masson V, Gerard RD, Gils A, Carmeliet G, Carmeliet P, Declerck PJ, Noel A, Foidart JM (2002) The pro- or antiangiogenic effect of plasminogen activator inhibitor 1 is dose dependent. FASEB J 16: 147-154

Duffy MJ (1996) Proteases as prognostic markers in cancer. Clin Cancer Res 2: 613-618

Duffy MJ, Maguire TM, McDermott EW, O’Higgins N (1999) Urokinase plasminogen activator: a prognostic marker in multiple types of cancer. $J$ Surg Oncol 71: 130-135

Fischerman K, Mouridsen HT (1988) Danish Breast Cancer Cooperative Group (DBCG). Structure and results of the organization. Acta Oncol 27: 593-596

Folkman J (1971) Tumor angiogenesis: therapeutic implications. N Engl J Med 285: 1182-1186

Folkman J (1995) Tumor angiogenesis. In The Molecular Basis of Cancer, Mendelsohn J, Howley PM, Israel MA, Liotta LA (eds) pp 206-232. Philadelphia: W.B. Saunders.

Fox SB (1997) Tumour angiogenesis and prognosis. Histopathology 30: 294-301

Fox SB, Leek RD, Weekes MP, Whitehouse RM, Gatter KC, Harris AL (1995) Quantitation and prognostic value of breast cancer angiogenesis: comparison of microvessel density, Chalkley count, and computer image analysis. J Pathol 177: 275-283

Fox SB, Taylor M, Grondahl-Hansen J, Kakolyris S, Gatter KC, Harris AL (2001) Plasminogen activator inhibitor-1 as a measure of vascular remodelling in breast cancer. J Pathol 195: 236-243

Hansen S, Grabau DA, Rose C, Bak M, Sørensen FB (1998) Angiogenesis in breast cancer. A comparative study of the observer variability of methods for determining microvessel density. Lab Invest 78: 1563-1573

Hansen S, Grabau DA, Sørensen FB, Bak M, Vach W, Rose C (2000a) Vascular grading of angiogenesis: prognostic significance in breast cancer. Br J Cancer 82: 339-347
Hansen S, Grabau DA, Sørensen FB, Bak M, Vach W, Rose C (2000b) The prognostic value of angiogenesis by Chalkley counting in a confirmatory study design on 836 breast cancer patients. Clin Cancer Res 6: 139-146

Harbeck N, Graeff H, Hofler H, Brunner N, Schmitt M (1998) Plasminogen activator system in breast cancer tissue and blood: clinical relevance and methodological considerations. J Tumor Marker Oncol 13: 19-35

Harrell Jr FE, Lee KL, Matchar DB, Reichert TA (1985) Regression models for prognostic prediction: advantages, problems, and suggested solutions. Cancer Treat Rep 69: 1071-1077

Hildenbrand R, Dilger I, Horlin A, Stutte HJ (1995) Urokinase and macrophages in tumour angiogenesis. $\mathrm{Br} J$ Cancer 72: 818-823

Janicke F, Prechtl A, Thomssen C, Harbeck N, Meisner C, Untch M, Sweep CG, Selbmann HK, Graeff H, Schmitt M (2001) Randomized adjuvant chemotherapy trial in high-risk, lymph node-negative breast cancer patients identified by urokinase-type plasminogen activator and plasminogen activator inhibitor type 1. J Natl Cancer Inst 93: 913-920

Kaplan EL, Meier P (1958) Nonparametric estimation from incomplete observations. J Am Stat Assoc 53: 457-481

Knoop A, Andreasen PA, Andersen JA, Hansen S, Lænkholm A-V, Simonsen ACW, Andersen J, Overgaard J, Rose C (1998) Prognostic significance of urokinase-type plasminogen activator and plasminogen activator inhibitor-1 in primary breast cancer. Br J Cancer 77: 932-940

Lambert V, Munaut C, Noel A, Frankenne F, Bajou K, Gerard R, Carmeliet P, Defresne MP, Foidart JM, Rakic JM (2001) Influence of plasminogen activator inhibitor type 1 on choroidal neovascularization. FASEB J 15: 1021-1027

Look MP, Foekens JA (1999) Clinical relevance of the urokinase plasminogen activator system in breast cancer. APMIS 107: 150-159

Look MP, van Putten WL, Duffy MJ, Harbeck N, Christensen IJ, Thomssen C, Kates R, Spyratos F, Ferno M, Eppenberger-Castori S, Sweep CG, Ulm K, Peyrat JP, Martin PM, Magdelenat H, Brunner N, Duggan C, Lisboa BW, Bendahl PO, Quillien V, Daver A, Ricolleau G, Meijer-van Gelder ME, Manders P, Fiets WE, Blankenstein MA, Broet P, Romain S, Daxenbichler G, Windbichler G, Cufer T, Borstnar S, Kueng W, Beex LV, Klijn JG, O’Higgins N, Eppenberger U, Janicke F, Schmitt M, Foekens JA (2002) Pooled analysis of prognostic impact of urokinase-type plasminogen activator and its inhibitor PAI-1 in 8377 breast cancer patients. J Natl Cancer Inst 94: 116-128

Mazar AP, Henkin J, Goldfarb RH (1999) The urokinase plasminogen activator system in cancer: implication for tumor angiogenesis and metastasis. Angiogenesis 3: 15-32

McMahon GA, Petitclerc E, Stefansson S, Smith E, Wong MK, Westrick RJ, Ginsburg D, Brooks PC, Lawrence DA (2001) Plasminogen activator inhibitor-1 regulates tumor growth and angiogenesis. J Biol Chem 276: 33964-33968

Mignatti P, Rifkin DB (1996) Plasminogen activators and matrix metalloproteinases in angiogenesis. Enzyme Protein 49: 117-137

Peduzzi P, Concato J, Kemper E, Holford TR, Feinstein AR (1996) A simulation study of the number of events per variable in logistic regression analysis. J Clin Epidemiol 49: 1373-1379

Pepper MS, Montesano R, Mandriota SJ, Orci L, Vassalli JD (1996) Angiogenesis: a paradigm for balanced extracellular proteolysis during cell migration and morphogenesis. Enzyme Protein 49: 138-162

Rose C, Hansen S, Grabau DA, Bak M, Vach W, Sørensen FB (2000) Prognostic value of angiogenesis by microvessel density in breast cancer patients. Proc Am Soc Clin Oncol 2580:

Scharff RW, Torloni H (1998) Histological typing of breast tumours. International histological classification of tumours, No.2. Geneva

Simon R, Altman DG (1994) Statistical aspects of prognostic factor studies in oncology. Br J Cancer 69: 979-985 